\title{
Clinico-Pathological Findings of Bangladeshi Covid 19 Patients with their Clinical Outcome: Study of A Cohort of 201 Cases
}

\author{
NU AHMED ${ }^{\mathrm{a}}$, MA ISLAM $^{\mathrm{b}}$, MA KABIR $^{\mathrm{c}}$, MH RAHMAN $^{\mathrm{d}}$, SMA SADAT $^{\mathrm{e}}$
}

\begin{abstract}
:
Introduction: COVID-19 is a major threat to human beings. Clinical characterization, rapid identification of cases and isolation are vital for containments of rapidly spreading disease. The objectives of the study were to evaluate the clinico pathologic profile of Covid 19 positive Bangladeshi patients and also to see their clinical outcome within defined period.

Methods: This cohort study on 201 Bangladeshi cases was done in Combined Military Hospital, a tertiary level hospital in Dhaka, Bangladesh from April 2020 to May 2020. Total 201 COVID-19 cases were enrolled after getting the result positive for RT-PCR. After collection, data were analysed to show the characteristics of Covid 19 and their outcome after treatment.

Results: Among 201 cases, 180 (90\%) were male and 21 (10\%) were female. The most prevalent affected age groups were $71(35.5 \%)$ patients in 26-35 years age, 54 (27\%) in 1625 years, 49 (24.5\%) in 35-45 years. Mean age is 32.2 \pm 2 . Among the total cases, 146 (73\%) have positive history of contact, 37 (18.5\%) have no history of any contact, 8 (4\%) denied any contact with COVID-19 patients. Regarding clinical presentations, 67 (33.5\%) patients presented with only one symptoms, 125 (62.5\%) had multiple symptoms
\end{abstract}

Introduction:

Coronaviruses have long been recognized as important pathogens that infect the respiratory tracts of domestic

a. Lt Col Dr. Nasir Uddin Ahmed, Classified Medicine Specialist and Endocrinologist, $\mathrm{CMH}$, Dhaka, Bangladesh

b. Maj General Dr. Md. Azizul Islam, OJT Medical Oncology ( Pakistan and Singapore), Professor Medicine and Medical Oncology, Consultant Physician General, DGMS. Bangladesh

c. Brig General Dr. Md. Anwarul Kabir, Adviser Spl in Medicine, CMH, Dhaka, Bangladesh

d. Brigadier General Dr. Md. Habibur Rahman, Professor of Medicine, Armed Forces Medical College, Dhaka, Bangladesh

e. S. M. Anwar Sadat, Associate Professor, Dept of Oral and Maxillofacial Surgery, Dhaka Dental College, Mirpur-14, Dhaka, Bangladesh.

Address of Correspondence: Lt Col Dr. Nasir Uddin Ahmed, Classified Medicine Specialist and Endocrinologist, CMH, Dhaka, Bangladesh.

Cell:+8801819086505, E-mail: nasir_amc@yahoo.com and $9(4.5 \%)$ cases were asymptomatic. 154 (77\%) patients presented with fever. Other presentations were cough 71 (35.5\%), headache 27 (13.5\%), myalgia 25 (12.5\%), sore throat 25 (12.5\%), malaise 15 (7.5\%), respiratory distress 11 (5.5\%). Respiratory system was the dominant domain of clinical presentation. Leukopenia was presented by 12 patients and 12 had lymphopenia. 18 patients had mild thrombocytopenia. Pulse oxymetry showed oxygen saturation below $88 \%$ in 12 cases. After oxygen therapy 7 cases were improved and 5 cases were shifted to Corona ICU as their saturation fell below 70. These 5 patients are categorised as severe disease, rest 196 patients were mild in nature.

Conclusion: COVID 19 affects male more than female. Common symptoms are fever, cough, headache, myalgia, sore throat, malaise, respiratory distress. Respiratory system is the dominant domain of clinical presentation. ICU support was needed in $2.5 \%$ cases and death rate was $1 \%$ which was associated with comorbidity of CKD.

Key Words: Bangladeshi patients, COVID 19, Cohort study, RT PCR.

(J Bangladesh Coll Phys Surg 2020; 38: 37-42) DOI: https://doi.org/10.3329/jbcps.v38i0.47346

and companion animals and are the causes of mild and severe respiratory diseases in humans. ${ }^{1,2}$ Coronaviruses are enveloped single-stranded RNA viruses that are zoonotic in nature. ${ }^{3}$ Although most human coronavirus infections are mild, the epidemics of the two betacoronaviruses, severe acute respiratory syndrome coronavirus (SARS-CoV) ${ }^{4-6}$ and Middle East respiratory syndrome coronavirus (MERS-CoV), ${ }^{7,8}$ have caused more than 10000 cumulative cases in the past two decades, with mortality rates of $10 \%$ for SARS-CoV and $37 \%$ for MERS-CoV. ${ }^{9}, 10$ On 31 st December 2019,27 cases of pneumonia of unknown aetiology were identified in Wuhan City, Hubei province in China. ${ }^{11}$ The causative agent was identified from throat swab samples conducted by the Chinese Centre for Disease Control and Prevention (CCDC) on 7th January 2020, and was subsequently named Severe Acute Respiratory Syndrome Coronavirus 2 (SARS-CoV-2). The disease was named COVID-19 by the World Health Organization 
(WHO). ${ }^{12}$ COVID-19 is mainly transmitted through respiratory droplets and contact. ${ }^{13}$ At present, patients with COVID-19 are the main source of infection. ${ }^{14}$ What's more remarkable is that asymptomatic infections can also be a source of infection. ${ }^{15}$ Around $80 \%$ of COVID19 infections present as a mild respiratory illness in a patient who is ambulatory and can generally be managed outside the hospital. Around 15\% typically need hospital care (usually for moderate to severe pneumonia), and another 5\% have critical illness requiring more intensive supports. ${ }^{16}$

Studies have shown that COVID-19 could induce fever, dry cough, dyspnea, and fatigue in infected patients. In more severe cases, infections caused viral pneumonia and could lead to severe acute respiratory distress syndrome (ARDS) and even death. ${ }^{17}$ Pharyngodynia, nasal congestion, and rhinorrhoea have been reported in patients with COVID-19. ${ }^{18}$

In this study, the clinical characteristics of 201 patients diagnosed and admitted with COVID-19 in Dhaka Combined Military Hospital were evaluated. The objectives of the study were to evaluate the clinico pathologic profile of Covid 19 positive Bangladeshi patients and also to see their clinical outcome within defined period.

\section{Materials and method:}

Study design and participants

This cohort study was conducted in $\mathrm{CMH}$, Dhaka, a tertiary level hospital, Bangladesh from April 2020 to May 2020. We selected consecutive 201 patients from a specific corona dedicated isolation ward of this hospital. We applied 2 inclusion criteria: (i) all patients were confirmed by real-time polymerase chain reaction and were diagnosed as having COVID-19 according to WHO interim guidance and (ii) all patients who underwent chest X-ray and complete panel of routine laboratory tests, including complete blood count, blood biochemistry, serum LDH, D-dimer, Ferritin and procalcitonin. Patients who did not meet the above inclusion criteria were excluded from our study.

\section{Procedures}

The demographics data, clinical characteristics, laboratory data, treatment programs, and outcome measures were recorded prospectively. Throat swab specimens from the upper respiratory tract obtained from all patients at admission were immediately maintained in a viral transport medium and were tested to confirm COVID-19 by real-time polymerase chain reaction.

\section{Outcome data}

We extracted the history (i.e., clear contact history and unclear contact history), demographic data, clinical characteristics including symptoms on admission and co-morbidities, laboratory data, treatment programs, and clinical outcomes. All the data were collected in predefined data collection sheet and statistical analyses were performed in SPSS Version 17.

\section{Results:}

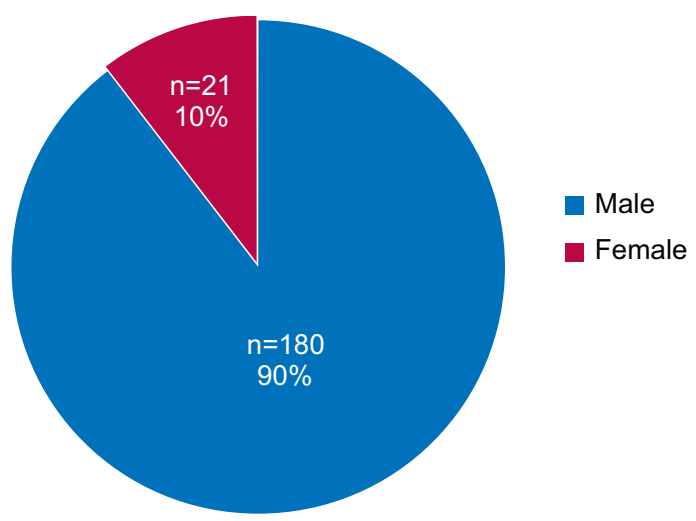

Fig.-1: shows sex distribution of cases $(n=201)$ : Male were 180 (90\%) and Female were 21 (10\%).
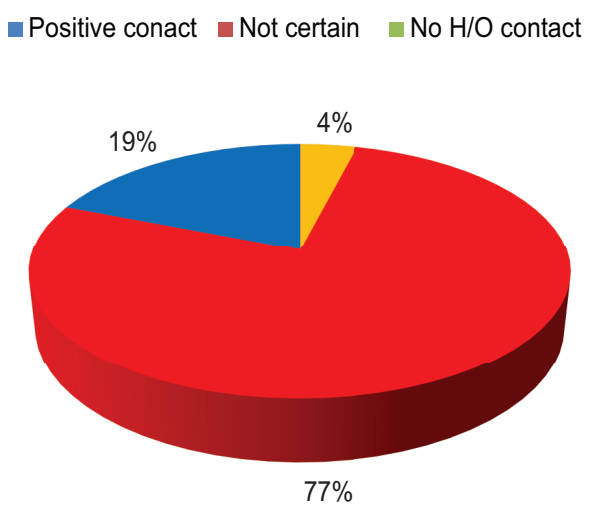

Fig.-2: shows history of contact with COVID-19 patients 146 (76\%), not aware of contact 37 (19\%) and no history of contact $8(4 \%)(n=201)$. 


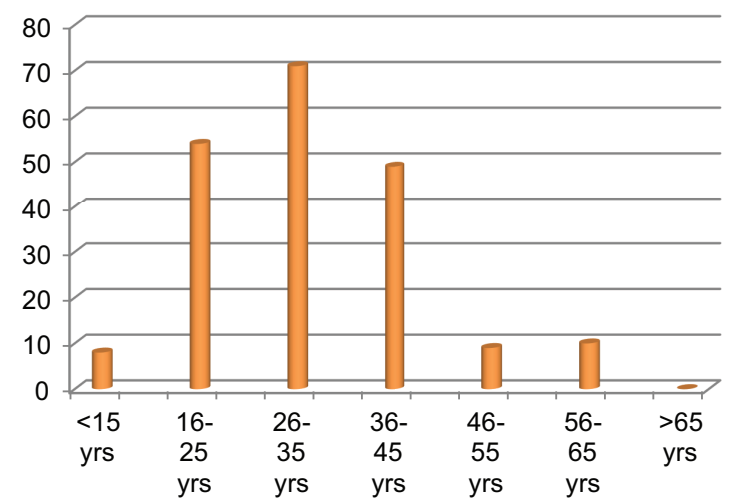

Fig.-3: Shows Age Distribution of COVID-19 Patients $(n=201)$.

Here 8 patients ( $4 \%$ ) were below 15 years, 54 (27\%) were in $16-25$ years, $71(35.5 \%)$ were in $26-35$ years, $49(24.5 \%)$ were in $35-45$ years, $9(4.5 \%)$ were in $46-55$ in years, 10 $(5 \%)$ were in $56-65$ years age group.

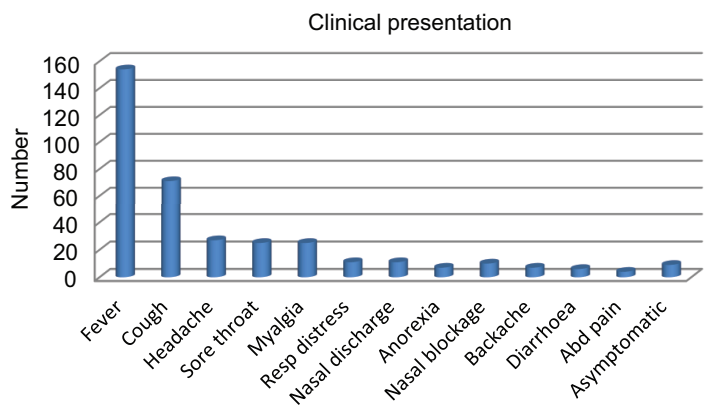

Fig.-4: shows clinical presentation of COVID-19 patients $(n=201)$

Here shows symptoms of fever 154 , cough 71 , headache 27 , sore throat 25 , myalgia 25 , respiratory distress 11 , nasal discharge 11 , anorexia 7 , nasal blockage 10 , backache 7, diarrhoea 6 patients.

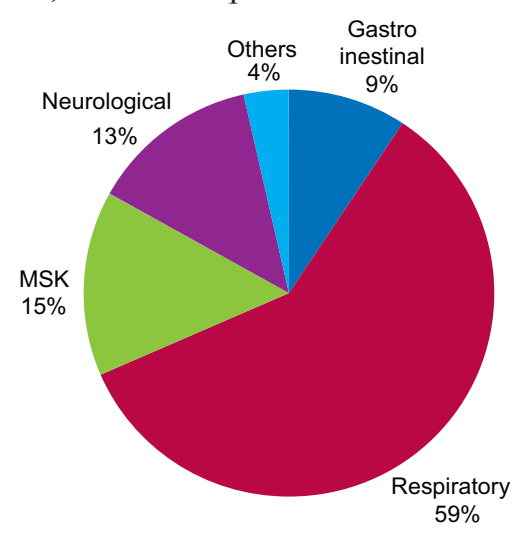

Fig.-5: shows system wise presentation of COVID-19 patients of which respiratory symptoms were $59 \%$, MSK 15\%, Neurological 13\%, Gastrointestinal 9\% and others $4 \% .(n=201)$
Table-I

\begin{tabular}{lc}
\multicolumn{2}{c}{ Clinical parameters and their values (in mean) } \\
Parameter & Values in mean \\
\hline Pulse & $84 \pm$ SD9.82 \\
SBP & $115 \pm$ SD 10.11 \\
DBP & $74 \pm$ SD5.90 \\
Temp & $100 \pm S D 1.39$ \\
R/R & $19 \pm$ SD1.26 \\
\hline
\end{tabular}

Total 201 COVID-19 patients were enrolled. Among them $180(90 \%)$ were male and $21(10 \%)$ female. Most patients were from 26-35 years age group which was 71 (35.5\%); then from $16-25$ years age group 54 (27\%), from $35-45$ years $49(24.5 \%), 8$ patients (4\%) below 15 years, 9 (4.5\%) from $46-55$ years, 10 (5\%) from 56-65 yrs. Mean age is $32.2 \pm 2$. Among the patients 21 had multiple comorbidities like DM, HTN, IHD, ESRD ON MHD, bronchial asthma and pheochromocytoma. Among 201 patients 146 (73\%) have positive history of contact with COVID-19 patients, 37 (18.5\%) not aware of any contact. Only 8 (4\%) patients denied any contact with COVID19 patients. Regarding clinical presentation, 67 (33.5\%) patients presented with only one symptoms, 125 (62.5\%) had multiple symptoms, 9 (4.5\%) were asymptomatic. Fever was the dominant presenting feature. 154 (77\%) patients presented with fever. Other presentations were cough $71(35.5 \%)$, headache $27(13.5 \%)$, myalgia 25 (12.5\%), sore throat $25(12.5 \%)$, malaise $15(7.5 \%)$, respiratory distress $11(5.5 \%)$. Rare symptoms were nasal obstruction (10), backache (7), diarrhoea (6), abdominal pain (4), chest pain (4), palpitation (3), burning whole body (2), and toothache (2). Oral ulcer, constipation, red eye, fall, thigh pain were also the presenting feature of each of 1 patient. Respiratory system was the dominant domain of clinical presentation. 133 patients had respiratory symptoms, 33 have musculoskeletal symptoms, 30 had neurological symptoms and 21 had gastro intestinal features.

Mean pulse were $84.62 \pm$ SD9.82 bpm, mean SBP 115.72 \pm SD10.11 mm of Hg, mean DBP 74.63 \pm SD5.908 $\mathrm{mm}$ of $\mathrm{Hg}$, mean temperature $100.27 \pm \mathrm{SD} 1.39 \mathrm{~F}$, respiratory rate $19.17 \pm \mathrm{SD} 1.26 / \mathrm{m}$.

Blood picture showed mean $\mathrm{Hb} 14.20 \pm \mathrm{SD} 1.46 \mathrm{gm} / \mathrm{dl}$, TLC $5.09 \pm \mathrm{SD} 1.59 \times 10^{9}$, mean Neutrophil count $54.94 \pm \mathrm{SD} 10.34 \times 10^{9}$, mean Lymphocyte count 
$36.29 \pm$ SD9.68 $\times 10^{9}$, mean Platelet count $229.22 \pm$ SD184.47 $\times 10^{9}$. Leucopenia were presented by 12 patients and 12 had lymphopenia. 18 patients have mild thrombocytopenia.

Oxygen saturations were measured by pulse oximetry showed 12 patients below $88 \%$. After oxygen therapy all improved except 5 who were shifted to Corona ICU as their saturation fell below 70 . These 5 patients were categorised as severe disease, rest 196 patients were mild in nature. Their chest X-ray had no evidence of pneumonia. ICU support was needed in $2.5 \%$ cases and death rate was $1 \%$ which was associated with comorbidity of CKD.

\section{Discussion:}

In our study there was significant difference in the proportion of male and female patients, and infection in children was rare, which was consistent with the results of a study performed by Zhong et al. ${ }^{19}$ Their results showed that males were more likely to be infected than females. ${ }^{19}$

A total of $73 \%$ of the patients included in this study had contact history which again verified the conclusion of human to human transmission. ${ }^{20}$ Some COVID-19 cases had atypical symptoms or were asymptomatic (4.5\%). Furthermore, asymptomatic persons are potential sources of SARS-CoV-2 transmission ${ }^{21}$. It appears that transmission is possible during the incubation period, and the carrier cannot be spotted. ${ }^{21}$ The age group mostly affected were $26-35$ years (35.5\%). In 16-24 years age group (27\%), in 36-45 age groups (24.5\%), in 56-56 year age group $5 \%$, in $46-55$ years age group $4.5 \%$ were affected. Youngest patient was 2 years 6 months of age. Mean age 32.2 $\pm \mathrm{SD} 2$. In our study fever was the dominant symptoms. $154(77 \%)$ patients presented with fever which was consistent with Guan et al, ${ }^{22}$ Wang et al, ${ }^{23}$ Zangh et al, ${ }^{24}$ Next to fever cough was also prominent presenting feature $(35.5 \%)$ which was also consistent with other research findings. ${ }^{22-24}$

A systematic review by Rodriguez-Morales et al ${ }^{25}$ of data on 656 cases published in January and February 2020 reported fever in $88.7 \%$, cough in $57.6 \%$, dyspnoea in $45.6 \%$, myalgia or fatigue in $29.4 \%$, sore throat in $11.0 \%$, headache in $8.0 \%$, and diarrhoea in $6.1 \%$. In our study sore throat was $(12.5 \%)$, myalgia (12.5\%), fatigue (7.5\%) diarrhoea (3\%) also consistent with that findings though headache (13.5\%) little higher than that.
On March 22, 2020, the American Academy of Otolaryngology - Head and Neck Surgery stated that anosmia and dysgeusia have been reported by patients ultimately testing positive for SARS-CoV-2 and proposed to add these symptoms to the list of screening tools for possible COVID-19 infection. ${ }^{26}$ In our study there were 5 patients $(2.5 \%)$ whose initial complaints included anosmia or dysgeusia. Very few patients were presented with atypical symptoms like burning body $(1 \%)$, toothache $(1 \%)$, itchiness $(0.5 \%)$, red eye $(0.5 \%)$, oral ulcer $(0.5 \%)$, constipation $(0.5 \%)$ and fall $(0.5 \%)$. In our study 21 patients having multiple co-morbidities which included DM, HTN, CKD on MHD, IHD, bronchial asthma, pheochromocytoma. 3 patients were on maintenance haemo-dialysis and so shifted to corona dedicated dialysis unit in our centre. One patient was special child.

It is understandable that, faced with a devastating pandemic and a medical and societal imperative, there is great enthusiasm for promising news of treatments. Picking and supporting the best therapies and preventions to tackle the COVID-19 pandemic head on is one of the scientific community's most urgent priorities. ${ }^{27}$ Though there was no specific therapy against this virus but in this study group of patients were treated with protocol of treatment made by hospital which included antipyretics, antihistamine, supplemental Vitamin-C, Zinc, high calorie diet, oxygen supplementation where needed. All the patients were given either Hydroxychloroquine, Doxycycline, Azythromycin or Favipiravir alone or in combination accordingly. D-dimer positive patients were given enoxaperin. All patients were discharged 10 days course of treatment. Some groups were discharged even early. Discharge criteria were two negative RT-PCR 24 hours apart. 5 patients were shifted to ICU out of them 2 patients died. One of them was admitted in critical state with history of CKD on MHD, not attending dialysis for 02 sessions. Fatal outcome was $1 \%$.

\section{Conclusion:}

Covid 19 affects male more than female. Common symptoms are fever, cough, headache, myalgia, sore throat, malaise, respiratory distress. Respiratory system is the dominant domain of clinical presentation. Rare symptoms were nasal obstruction, backache, diarrhoea, abdominal pain, chest pain, palpitation, burning whole 
body, toothache, Oral ulcer, constipation, red eye and thigh pain. Some cases show Leucopenia, lymphopenia and mild thrombocytopenia. Sometimes patients present with gradual lowering of oxygen saturation for which oxygen therapy according to the demand is needed and in rare situation ICU support becomes necessary. In this study, ICU support was needed in $2.5 \%$ cases and death rate was $1 \%$ which was associated with comorbidity of CKD.

The outcome of study needs to be further verified by large sample with multi-centre study. Extended followup would provide more detailed information about potential risk factors for the disease and the factors that would influence clinical outcomes.

\section{References:}

1. Su S,Wong G,Shi W,Liu J,Lai ACK,Zhou J.et al. Epidemiology,Genetic Recombination and Pathogenesis of Coronaviruses.Trends Microbiol.2016;24(6):490-502.

2. Weiss SR,Navas-Martin S.Coronavirus pathogenesis and the emerging pathogen severe acute respiratory syndrome coronavirus. Microbiol Mol Biol Rev.2005;69(4):635-64.

3. Zhu N, Zhang D, Wang W, Li X, Yang B, Song J.et al. A novel coronavirus from patients with pneumonia in China, 2019.N Engl J Med 2020;382(8):727-733

4. Ksiazek TG, Erdman D, Goldsmith CS,Zaki SR,Peret T,Emery S.et al. A novel coronavirus associated with severe acute respiratory syndrome. N Engl J Med 2003;348: 19531966.

5. Kuiken T, Fouchier RAM, Schutten M,Rimmelzwaan GF,Amerongen GV,Riel DV.et al. Newly discovered coronavirus as the primary cause of severe acute respiratory syndrome. Lancet 2003; 362: 263-270.

6. Drosten C, Günther S, Preiser W, Werf Svd,Brodt HR,Becker S.et al.Identification of a novel coronavirus in patients with severe acute respiratory syndrome. N Engl J Med 2003; 348: 1967-1976.

7. de Groot RJ, Baker SC, Baric RS, et al. Middle East respiratory syndrome coronavirus (MERS-CoV): announcement of the Coronavirus Study Group. J Virol 2013; 87: 7790-92.

8. Zaki AM, Van Boheemen S, Bestebroer TM, Osterhaus ADME, Fouchier RAM. Isolation of a novel coronavirus from a man with pneumonia in Saudi Arabia. N Engl J Med 2012;367:1814-1820.

9. WHO. Summary of probable SARS cases with onset of illness from 1 November 2002 to 31 July 2003. Dec 31, 2003. https://www. who.int/csr/sars/country/ table2004_04_21/en/ (accessed Jan 19, 2020).
10 WHO. Middle East respiratory syndrome coronavirus (MERS-CoV). November, 2019. http://www.who.int/ emergencies/mers-cov/en/ (accessed Jan 19, 2020). 9 WHO.

11. H.Lu,C.W Stratton,Y.Tang.Outbreak of pneumonia of unknown etiology in wuhan China: the mystery and the miracle, J. Med.Virol.2020;92(4):401-402.

12. World Health Organization, WHO Director-General's Remarks at the Media Briefing on 2019-nCoV on 11 February 2020, (2020) https://www.who.int/dg/speeches/ detail/who-director-general-s-remarks-at-the-mediabriefing-on-2019-ncov-on-11- february-2020.

13. Yang F,Liu N,Wu J Y. Pulmonary rehabilitation guidelines in the principle of $4 \mathrm{~S}$ for patients infected with 2019 novel coronavirus (2019-nCoV). Zhonghua Jie He He Hu Xi Za Zhi, 2020, 43: E004.

14. Chen Z-M,Fu J-F,Shu Q,Chen YH,Hua CZ,Li FB.et al.Diagnosis and treatment recommendations for pediatric respiratory infection caused by the 2019 novel coronavirus. World J Pediatr.2020;16:240-246.doi:10.1007/s12519020-00345-5.

15. Tuite Ashleigh R,Fisman David N,Reporting.Epidemic Growth, and Reproduction Numbers for the 2019 Novel Coronavirus (2019-nCoV) Epidemic. Ann. Intern. Med., 2020;172(8):567-568. doi:10.7326/M20-0358.

16. Wu Z, McGoogan JM. Characteristics of and Important Lessons From the Coronavirus Disease 2019 (COVID-19) Outbreak in China. JAMA. 2020;323(13):1239-1242.

17. Guan WJ,Ni ZY,Hu Y,Liang WH,Ou CQ,He JX.et al. Clinical characteristics of coronavirus disease 2019 in China [published online ahead of print 2020]. N Engl J Med.2020;382:1708-1720. doi: 10.1056/ NEJMoa2002032.

18. Chen N, Zhou M, Dong X, Y,Qu J,Gong F,Han Y et al. Epidemiological and clinical characteristics of 99 cases of 2019 novel coronavirus pneumonia in Wuhan, China: a descriptive study. Lancet. 2020;395(10223): 507-513. doi: 10.1016/S0140-6736(20)30211-7.

19. Guan W, Ni Z, Hu Y, Liang W, Ou C, He J, Liu L. Clinical Characteristics of 2019 Novel Coronavirus Infection in China. N Engl J Med 2020; 382:1708-1720.

20. Phan LT, Nguyen TV, Luong QC, Nguyen TV, Nguyen HT, Le HQ, et al. Importation and human to human transmission of a novel coronavirus in Vietnam. N Engl J Med. 2020;382:872 874.

21. Rothe C, Schunk M, Sothmann P, Bretzel G, Froeschl G, Wallrauch C, et al. Transmission of 2019-nCoV infection from an asymptomatic contact in Germany. New England Journal of Medicine N 2020;382:970-971doi: 10.1056/ NEJMc2001468.

22. Guan WJ, Ni ZY, Hu Y, et al. Clinical characteristics of coronavirus disease 2019 in China. N Engl J Med 2020; 382:1708-1720 
23. Wang D, Hu B, Hu C, Zhu F,Liu X,Zang J,et al. Clinical characteristics of 138 hospitalized patients with 2019 novel coronavirus-infected pneumonia in Wuhan, China [published online ahead of print 2020]. JAMA. 2020Mar 17;323(11):1061-1069.doi: 10.1001/jama.2020.1585.

24. Zhang JJ, Dong X, Cao YY,Yuan YD, Yang YB, Yan YQ. et al. Clinical characteristics of 140 patients infected with SARS-CoV-2 in Wuhan, China [published online ahead of print 2020]. Allergy.2020 Feb 19[cited.....] Available https://doi.org/10.1111/all.14238

25. Rodriguez-Morales AJ, Cardona-Ospina JA, GutiérrezOcampo E, Penna RV,Holguin-Rivera Y,Escalera Antezana JPet al. Clinical, laboratory and imaging features of COVID-
19: a systematic review and meta-analysis. Travel Med Infect Dis 2020;34:101623. doi: 10.1016/ j.tmaid.2020.101623 [Epub ahead of print]

26. Anosmia-hyposmia-anddysgeusia-symptoms-coronavirusdisease AAO-HNS Covid 19 resouces.[Internet]2020 March 22 [cited March 25, 2020.]Available https:// www.entnet.org/content/aao-hns-anosmia-hyposmiaanddysgeusia-symptoms-coronavirus-disease

27. BrayM., Rayner C., Noël F., Jans D., Wagstaff K., Ivermectin and COVID-19: a report in Antiviral Research, widespread interest, an FDA warning, two letters to the editor and the authors' responses, Antiviral Research, https:/ /doi.org/10.1016/j.antiviral.2020.104805 